\title{
NORMALITY IN GROUP RINGS
}

\author{
V. A. BOVDI AND S. SICILIANO
}

Dedicated to Professor P. M. Gudivok on the occasion of his 70th birthday

\begin{abstract}
Let $K G$ be the group ring of a group $G$ over a commutative ring $K$ with unity. The rings $K G$ are described for which $x x^{\sigma}=x^{\sigma} x$ for all $x=\sum_{g \in G} \alpha_{g} g \in K G$, where $x \mapsto x^{\sigma}=\sum_{g \in G} \alpha_{g} f(g) \sigma(g)$ is an involution of $K G$; here $f: G \rightarrow U(K)$ is a homomorphism and $\sigma$ is an antiautomorphism of order two of $G$.
\end{abstract}

Let $R$ be a ring with unity. We denote by $U(R)$ the group of units of $R$. A (bijective) map $\diamond: R \rightarrow R$ is called an involution if for all $a, b \in R$ we have $(a+b)^{\diamond}=a^{\diamond}+b^{\diamond}$, $(a b)^{\diamond}=b^{\diamond} \cdot a^{\diamond}$ and $a^{\diamond^{2}}=a$. Let $K G$ be the group ring of a group $G$ over a commutative ring $K$ with unity, let $\sigma$ be an antiautomorphism of order two of $G$, and let $f: G \rightarrow U(K)$ be a homomorphism from $G$ onto $U(K)$. For an element $x=\sum_{g \in G} \alpha_{g} g \in K G$, we define $x^{\sigma}=\sum_{g \in G} \alpha_{g} f(g) \sigma(g) \in K G$. Clearly, $x \mapsto x^{\sigma}$ is an involution of $K G$ if and only if $g \sigma(g) \in \operatorname{Ker} f=\{h \in G \mid f(h)=1\}$ for all $g \in G$.

The ring $K G$ is said to be $\sigma$-normal if

$$
x x^{\sigma}=x^{\sigma} x
$$

for each $x \in K G$. The properties of the classical involution $x \mapsto x^{*}$ (where $*: g \mapsto g^{-1}$ for $g \in G$ ) and the properties of normal group rings (i.e., $x x^{*}=x^{*} x$ for each $x \in K G$ ) have been used actively for the investigation of the group of units $U(K G)$ of the group ring $K G$ (see $[1,2]$ ). Moreover, they also have important applications in topology (see $[7,8])$. Our aim is to describe the structure of the $\sigma$-normal group ring $K G$ for an arbitrary order 2 antiautomorphism $\sigma$ of the group $G$. Note that descriptions of the classical normal group rings and the twisted group rings were obtained in $[1,3]$ and $[4,5]$, respectively.

The notation used throughout the paper is essentially standard. $C_{n}$ denotes the cyclic group of order $n ; \zeta(G)$ and $C_{G}(H)$ are the center of the group $G$ and the centralizer of $H$ in $G$, respectively; $(g, h)=g^{-1} h^{-1} g h=g^{-1} g^{h}(g, h \in G) ; \gamma_{i}(G)$ is the $i$ th term of the lower central series of $G$, i.e., $\gamma_{1}(G)=G$ and $\gamma_{i+1}(G)=\left(\gamma_{i}(G), G\right)$ for $i \geq 1 ; \Phi(G)$ denotes the Frattini subgroup of $G$. We say that $G=A$ Y $B$ is a central product of its subgroups $A$ and $B$ if $A$ and $B$ commute elementwise and, taken together, they generate $G$, provided that $A \cap B$ is a subgroup of $\zeta(G)$.

A non-Abelian 2-generated nilpotent group $G=\langle a, b\rangle$ with an antiautomorphism $\sigma$ of order 2 is called a $\sigma$-group if $G^{\prime}$ has order $2, \sigma(a)=a(a, b)$, and $\sigma(b)=b(a, b)$.

Our main result reads as follows.

Theorem. Let $K G$ be the noncommutative group ring of a group $G$ over a commutative ring $K$ and $f: G \rightarrow U(K)$ a homomorphism. Assume that $\sigma$ is an antiautomorphism of order two of $G$ such that $x \mapsto x^{\sigma}$ is an involution of $K G$. Put $\mathfrak{R}(G)=\{g \in G \mid \sigma(g)=g\}$.

2000 Mathematics Subject Classification. Primary 16S34.

Key words and phrases. Group ring, normality.

This research was supported by OTKA no. T 037202 and no. T 038059. 
The group ring $K G$ is $\sigma$-normal if and only if $f: G \rightarrow\{ \pm 1\}, G, K$, and $\sigma$ satisfy one of the following conditions:

(i) $G$ has an Abelian subgroup $H$ of index 2 such that $G=\langle H, b\rangle, f(b)=-1, f(h)=1$, $\sigma(b)=b$, and $\sigma(h)=b^{-1} h b=b h b^{-1}$ for all $h \in H$;

(ii) $G=H Y \mathfrak{C}$ is a central product of a $\sigma$-group $H=\langle a, b\rangle$ and an Abelian group $\mathfrak{C}$ such that $G^{\prime}=\left\langle c \mid c^{2}=1\right\rangle$ and $H \subset \operatorname{Ker}(f)$. Moreover, either $\sigma(d)=d$ for all $d \in \mathfrak{C}$, $\mathfrak{C} \subset \operatorname{Ker}(f), \mathfrak{R}(G)=\zeta(G)$, and

$$
G / \mathfrak{R}(G)=\langle a \zeta(G), b \zeta(G)\rangle \cong C_{2} \times C_{2},
$$

or $\mathfrak{R}(G)$ is of index 2 in $\zeta(G)$ and

$$
G / \mathfrak{R}(G)=\langle g \mathfrak{R}(G), h \mathfrak{R}(G), d \mathfrak{R}(G)\rangle \cong C_{2} \times C_{2} \times C_{2},
$$

where $d \in \mathfrak{C}, \sigma(d)=d c$, and $f(d)=-1$;

(iii) $\operatorname{char}(K)=2, G=S Y \mathfrak{C}$ is a central product of $S=Y_{i=1}^{n} H_{i}$ and an Abelian group $\mathfrak{C}$ such that $H_{i}=\left\langle a_{i}, b_{i}\right\rangle$ is a $\sigma$-group and $G=\operatorname{Ker}(f)$. Moreover, $G^{\prime}=\left\langle c \mid c^{2}=1\right\rangle, n \geq 2$, where $n$ is not necessarily a finite number, $\sigma\left(a_{i}\right)=a_{i} c, \sigma\left(b_{i}\right)=b_{i} c$ for all $i=1,2, \ldots$, and $\exp (G / \mathfrak{R}(G))=2$.

Furthermore, if $n$ is finite, then either $\sigma(d)=d$ for all $d \in \mathfrak{C}$ and

$$
G / \mathfrak{R}(G)=\chi_{i=1}^{n}\left\langle a_{i} \zeta(G), b_{i} \zeta(G)\right\rangle \cong \chi_{i=1}^{2 n} C_{2},
$$

or $\mathfrak{R}(G)$ is of index 2 in $\zeta(G)$ and

$$
G / \mathfrak{R}(G)=\chi_{i=1}^{n}\left\langle a_{i} \mathfrak{R}(G), b_{i} \mathfrak{R}(G)\right\rangle \times\langle d \mathfrak{R}(G)\rangle \cong \chi_{i=1}^{2 n+1} C_{2},
$$

where $d \in \mathfrak{C}$ and $\sigma(d)=d c$.

Note that, in parts (i) and (ii) of the theorem, the group $\mathfrak{C}$ may be equal to 1 .

To make the statements less cumbersome, in what follows we shall often talk of $\sigma$ normal group rings $K G$ without specifying the homomorphism $f: G \rightarrow U(K)$ and the antiautomorphism $\sigma$ of order two of $G$. In order to prove the main theorem, we need some preliminary lemmas.

Lemma 1. Let $U(R)$ be the group of units of the ring $R$, and let $x \mapsto x^{\diamond}$ be an involution of $R$. Suppose that $x x^{\diamond}=x^{\diamond} x$ for all $x \in R$. If $a \in U(R)$, then $a^{\diamond}=t a$, at $=t a$, and $t^{\diamond}=t^{-1}$, where $t \in U(R)$.

Proof. Clearly $a^{\diamond}=a t$ for some $t \in U(R)$, and $a^{\diamond} a=a a^{\diamond}$ implies that ata $=a^{2} t$ and $a t=t a$. Now $a=a^{\diamond^{2}}=(a t)^{\diamond}=t^{\diamond} a t=t^{\diamond} t a$, whence $t^{\diamond}=t^{-1}$.

Lemma 2. Let $K$ be a commutative ring, let $H=\langle a, b\rangle$ be a non-Abelian 2-generated subgroup of a group $G$, and let $f: G \rightarrow U(K)$ be a homomorphism. If the group ring $K G$ is $\sigma$-normal, then $f: H \rightarrow\{ \pm 1\}$ and one of the following conditions is fulfilled:

(i) $f(a)=1, f(b)=-1, \sigma(a)=(a, b) a, \sigma(b)=b,\left(b^{2}, a\right)=1,(a b)^{2}=(b a)^{2}$, $((a, b), a)=1$, and $((a, b), b)=(a, b)^{-2}$;

(ii) $f(a)=-1, f(b)=1, \sigma(a)=a, \sigma(b)=(a, b) b,\left(b, a^{2}\right)=1,(a b)^{2}=(b a)^{2}$, $((a, b), b)=1$, and $((a, b), a)=(a, b)^{-2}$;

(iii) $f(a)=f(b)=-1, \sigma(a)=a, \sigma(b)=b,\left(a^{2}, b\right)=\left(a, b^{2}\right)=1,(a b)^{2}=(b a)^{2}$, and $((a, b), a b)=1$;

(iv) $f(a)=f(b)=1, \sigma(a)=(a, b) a, \sigma(b)=(a, b) b$, and $\langle a, b\rangle$ is nilpotent of class 2 and such that $\gamma_{2}(\langle a, b\rangle)$ is of order 2 . 
Proof. Let $K G$ be a $\sigma$-normal ring. For any noncommutative $a, b \in G$ we can put $\sigma(a)=a t$ and $\sigma(b)=b s$, where $s, t \in G$. By Lemma 1 , at $=t a, b s=s b, \sigma(t)=t^{-1}$, and $\sigma(s)=s^{-1}$. Set $x=a+b \in K G$. Clearly, $x^{\sigma}=f(a) \sigma(a)+f(b) \sigma(b)$, and by (1) we have

$$
f(b) a \sigma(b)+f(a) b \sigma(a)=f(a) \sigma(a) b+f(b) \sigma(b) a .
$$

If $a \sigma(b)=b \sigma(a)=\sigma(a) b=\sigma(b) a$, then we get $s=t$ and $a b=b a$, a contradiction. Observe that if three of the elements $\{a \sigma(b), b \sigma(a), \sigma(a) b, \sigma(b) a\}$ coincide, then $s=t$ and $a b=b a$, a contradiction. We consider the following cases.

1. $a \sigma(b)=b \sigma(a)$. By (2), it follows that

$$
f(a)+f(b)=0, \quad a \sigma(b)=b \sigma(a), \quad \sigma(a) b=\sigma(b) a .
$$

2. $a \sigma(b)=\sigma(a) b$. This yields $a s b=a t b$, so that $s=t \in \zeta(H)$, and (2) ensures

$$
f(a)=f(b), \quad \sigma(a)=a t, \quad \sigma(b)=b t, \quad t \in \zeta(H) .
$$

3. $a \sigma(b)=\sigma(b) a$. Since $b \sigma(b)=\sigma(b) b$, we get $\sigma(b) \in \zeta(H)$, a contradiction.

Now put $x=a(1+b)$. Then $x^{\sigma}=(1+f(b) \sigma(b)) f(a) \sigma(a)$ and, by (1),

$$
f(a b) a \sigma(a b)+f(a) a b \sigma(a)=f(a) \sigma(a) a b+f(a b) \sigma(a b) a .
$$

We shall treat the following cases separately.

1. $a \sigma(a b)=a b \sigma(a)$. Formula (5) implies that

$$
f(b)=-1, \quad \sigma(b)=b, \quad(\sigma(a) a) \cdot b=b \cdot(\sigma(a) a) .
$$

2. $a \sigma(a b)=\sigma(a) a b$ and $a b \sigma(a)=\sigma(a b) a$. By (1) we have $a b=\sigma(b) a$ and $\sigma(b)=$ $a b a^{-1}$. Since $a \sigma(a b)=\sigma(a) a b$, we get $a b a^{-1} a t=a t b$ and $(b, t)=1$. Recall that $\sigma(b)=b s=s b$. So, by $(5)$,

$$
f(b)=1, \quad \sigma(b)=a b a^{-1}, \quad t \in \zeta(H), \quad s=\left(a^{-1}, b^{-1}\right)=\left(b, a^{-1}\right) .
$$

3. $a \sigma(b) \sigma(a)=\sigma(b) \sigma(a) a$. Then $a \sigma(b)=\sigma(b) a$ and $\sigma(b) \in \zeta(H)$, a contradiction.

Assume that (3) and (6) are true. Then $f(b)=-1, f(a)=1, \sigma(b)=b, \sigma(a)=$ $b^{-1} a b=b a b^{-1}$, whence $\left(b^{2}, a\right)=1$. Since $\sigma(a)=a\left(a^{-1} b^{-1} a b\right)=\left(b a b^{-1} a^{-1}\right) a$, we get $a^{-1} b^{-1} a b=b a b^{-1} a^{-1}$ and $(a b)^{2}=(b a)^{2}$. Obviously,

$$
b^{-1}(a, b) b=b^{-1}\left(b a b^{-1} a^{-1}\right) b=a b^{-1} a^{-1} b=a b a^{-1} b^{-1}=(a, b)^{-1},
$$

so that $((a, b), b)=(a, b)^{-2}$, and statement (i) of our lemma follows.

If (3) and (7) are fulfilled, then $f(b)=1, f(a)=-1, \sigma(a)=a, \sigma(b)=a^{-1} b a$, and $\left(a^{2}, b\right)=1$. Since $\sigma(b)=b\left(b^{-1} a^{-1} b a\right)=\left(a b a^{-1} b^{-1}\right) b$, we obtain $s=b^{-1} a^{-1} b a=$ $a b a^{-1} b^{-1}$ and $(a b)^{2}=(b a)^{2}$. Therefore, $\sigma(a)=a, \sigma(b)=a^{-1} b a,\left(a^{2}, b\right)=1$, and we arrive at statement (ii).

Assume (4) and (6). Then $f(a)=f(b)=-1, \sigma(a)=a, \sigma(b)=b$, and $\left(a^{2}, b\right)=1$. Moreover, $f(a b)=1$ and $\sigma(a b)=b a=a^{-1}(a b) a$. We put $x=b(1+a)$. Clearly, $x^{\sigma}=(a-1) b$, and (1) implies $b^{2} a+b^{2} a^{2}=a b^{2} a+a b^{2}$, whence $\left(b^{2}, a\right)=1$. Thus, statement (iii) of our lemma is fulfilled.

Finally, if (4) and (7) are true, then $f(a)=f(b)=1$ and $\left(b, a^{-1}\right) \in \zeta(H)$. Using the identity $(\alpha \beta, \gamma)=(\alpha, \gamma)(\alpha, \gamma, \beta)(\beta, \gamma)$, where $\alpha, \beta, \gamma \in G$, we see that $1=\left(a^{-1} a, b\right)=$ $\left(a^{-1}, b\right)(a, b)$, whence $s=\left(b, a^{-1}\right)=(a, b) \in \zeta(H)$ and $\sigma(a)=(a, b) a, \sigma(b)=(a, b) b$. Since $a=\sigma^{2}(a)$ and $(a, b) \in \zeta(H)$, we have $(a, b)^{2}=1$, which yields statement (iv). The proof is complete.

Lemma 3. Let $K G$ be a $\sigma$-normal group ring of a non-Abelian group $G$. Then $H=$ $\langle w \in G \mid \sigma(w) \neq w\rangle$ is a normal subgroup in $G$. If $H$ is Abelian, then $G$ satisfies statement (i) of the theorem. 
Proof. Set $W=\{w \in G \mid \sigma(w) \neq w\}$. Let $g \notin W$ be such that $g^{2} \notin \zeta(G)$. Then $\left(g^{2}, h\right) \neq 1$ and $(g, h) \neq 1$, respectively, for some $h \in G$.

We consider the following cases.

1. $\operatorname{char}(K) \neq 2$. Since $\sigma\left(g^{2}\right)=g^{2}$, we can use Lemma 2 for the group $\left\langle g^{2}, h\right\rangle$ to show that $-1=f\left(g^{2}\right)=( \pm 1)^{2}=1$, a contradiction.

2. $\operatorname{char}(K)=2$. Using Lemma 2 for $\langle g, h\rangle$, we get $\left(g^{2}, h\right)=1$, again a contradiction.

Thus, $g^{2} \in \zeta(G)$ for any $g \notin W$. Now, if $w \in W, g \in G \backslash W$, and $g^{-1} w g \notin W$, then $\sigma\left(g^{-1} w g\right)=g^{-1} w g$ and

$$
g^{-1} w g=\sigma\left(g^{-1} w g\right)=g \sigma(w) g^{-2} g=g^{-1} \sigma(w) g
$$

so that $\sigma(w)=w$, a contradiction. Therefore, $g^{-1} w g \in W$ and the subgroup $H=\langle W\rangle$ is normal in $G$.

Suppose that $H=\langle W\rangle$ is Abelian. If $a \in W$ and $c \in C_{G}(W) \backslash H$, then $c a \notin H$. Therefore, $c a=\sigma(c a)=\sigma(a) c$, whence $\sigma(a)=a$, a contradiction. This shows that $C_{G}(W)=H$ and for each $b \notin H$ there exists $w \in W$ such that $(b, w) \neq 1$.

We claim that if $b_{1}, b_{2} \in G \backslash H$, then $b_{1} b_{2} \in H$. The following cases will be treated separately:

1. $\operatorname{char}(K) \neq 2$ and $b_{1} b_{2} \in G \backslash H$. For each $b_{i}$ we choose $w_{i} \in W$ such that $\left(b_{i}, w_{i}\right) \neq 1$. By (i) or (ii) of Lemma 2 , in $\left\langle w_{i}, b_{i}\right\rangle$ we have $f\left(b_{i}\right)=-1$, so that $f\left(b_{1} b_{2}\right)=1$ and there exists $w \in W$ for which $\left(b_{1} b_{2}, w\right) \neq 1$. Since $\sigma\left(b_{1} b_{2}\right)=b_{1} b_{2}$, by (i) or (ii) of Lemma 2 we get $f\left(b_{1} b_{2}\right)=-1$, a contradiction.

2. $\operatorname{char}(K)=2$ and $b_{1} b_{2} \in G \backslash H$. Obviously, $b_{1} b_{2}=\sigma\left(b_{1} b_{2}\right)=b_{2} b_{1}$, whence $\left(b_{1}, b_{2}\right)=1$. Now, there is $w \in W$ with $\left(w, b_{1}\right) \neq 1$, and by Lemma 2 we get $\sigma(w)=$ $b_{1}^{-1} w b_{1}=b_{1} w b_{1}^{-1}$. Furthermore, $b_{1} b_{2} w \in G \backslash H$ and

$$
b_{1} b_{2} w=\sigma\left(b_{1} b_{2} w\right)=\sigma(w) b_{1} b_{2}=b_{1} w b_{2},
$$

implying $\left(b_{2}, w\right)=1$. Now $\left(b_{1}, b_{2} w\right)=\left(b_{1}, w\right) \neq 1$ and $b_{2} w \in G \backslash H$; applying Lemma 2 in $\left\langle b_{1}, b_{2} w\right\rangle$, we obtain $b_{2} w=\sigma\left(b_{2} w\right)=\sigma(w) b_{2}$ and $\sigma(w)=w$, a contradiction.

We have proved that $b_{1} b_{2} \in H$ for every $b_{1}, b_{2} \in G \backslash H$. Hence, $G=\langle H, b| b \notin H$, $\left.b^{2} \in H\right\rangle, f(b)=-1$, and $f(h)=1$ for all $h \in H$.

Finally, let $w \in W$ be such that $(b, w)=1$. Since $b \notin H=C_{G}(W)$, there exists $w_{1} \in W$ with $w_{1} \neq b^{-1} w_{1} b=\sigma\left(w_{1}\right)$. Clearly, we have $\left(w w_{1}, b\right) \neq 1$; using Lemma 2 for $\left\langle w w_{1}, b\right\rangle$, we obtain

$$
\sigma(w) \sigma\left(w_{1}\right)=\sigma\left(w_{1} w\right)=b^{-1} w_{1} w b=\sigma\left(w_{1}\right) w,
$$

whence $\sigma(w)=w$, a contradiction. Thus, $b^{-1} h b=\sigma(h)$ for all $h \in H$.

Lemma 4. Let $K G$ be a $\sigma$-normal group ring, let $W=\{w \in G \mid \sigma(w) \neq w\}$, and let $a, b \in W$ be such that $(a, b) \neq 1$. Put $\mathfrak{R}=\{g \in G \mid \sigma(g)=g\}$ and $\mathfrak{C}=C_{G}(\langle a, b\rangle)$. Then $\langle a, b\rangle$ is a $\sigma$-group, $\Phi(\langle a, b\rangle)=\zeta(\langle a, b\rangle)=\{g \in\langle a, b\rangle \mid \sigma(g)=g\}$, and

$$
\sigma(g)= \begin{cases}g & \text { if } g \in \zeta(\langle a, b\rangle), \\ g(a, b) & \text { if } g \notin \zeta(\langle a, b\rangle) .\end{cases}
$$

Moreover, $G=\langle a, b\rangle \mathrm{Y} \mathfrak{C}$, and either $\sigma(c)=(a, b) c$, or $\sigma(c)=c$, where $c \in \mathfrak{C}$. Also, the following is true:

(i) if $\mathfrak{C}$ is Abelian, then $G$ satisfies statement (ii) of the theorem;

(ii) if $\mathfrak{C}$ is not Abelian, then $\operatorname{char}(K)=2$.

Proof. Let $a, b \in W$ satisfy $(a, b) \neq 1$. By Lemma $2, f(a)=f(b)=1,\langle a, b\rangle$ is nilpotent of class 2 and such that $\left|\gamma_{2}(\langle a, b\rangle)\right|=2$, and $\sigma(a)=b^{-1} a b, \sigma(b)=a^{-1} b a$. Thus $\langle a, b\rangle$ is a $\sigma$-group. Any element $g \in\langle a, b\rangle$ can be written as $g=a^{i} b^{j}(a, b)^{k}$, where $i, j, k \in \mathbb{N}$. 
Since $\sigma(g)=g$, we conclude that $i$ and $j$ are even. Now by [6, Theorems 10.4 .1 and 10.4.3] we obtain

$$
\Phi(\langle a, b\rangle)=\zeta(\langle a, b\rangle)=\{g \in\langle a, b\rangle \mid \sigma(g)=g\} .
$$

Suppose $c \in W$ and $(a, c) \neq 1$. Again by Lemma $2,\langle a, c\rangle$ is nilpotent of class 2 and $\sigma(a)=c^{-1} a c=b^{-1} a b$, so that $(a, b)=(a, c)$. Now, let $c, d \in W$ be such that $(c, d) \neq 1$ and $\langle c, d\rangle \in \mathfrak{C}$. Obviously, $(a c, b)=(a, b) \neq 1$ and $(a c, d)=(c, d) \neq 1$. By Lemma 2, $\sigma(a c)=b^{-1}(a c) b=d^{-1}(a c) d$ and $(a, b)=(c, d)$, which shows that $H^{\prime}$ has order two and is central in $G$.

Let $g \in G \backslash \mathfrak{C} \cdot\langle a, b\rangle$. If $(a, g) \neq 1$, then, using Lemma 2 for $\langle a, g\rangle$, we get $\sigma(a)=$ $g^{-1} a g=b^{-1} a b$ and $(a, g)=(a, b)$. Similarly, if $(b, g) \neq 1$, then $(b, g)=(a, b)$.

The following cases are possible:

1. $(g, a)=1$ and $(g, b) \neq 1$. Then we have $(g a, b)=(g a, a)=1$, which implies that $g=(g a) \cdot a^{-1} \in \mathfrak{C} \cdot\langle a, b\rangle$.

2. $(g, a) \neq 1$ and $(g, b)=1$. Then we have $(g b, a)=(g b, b)=1$, which implies that $g=(g b) \cdot b^{-1} \in \mathfrak{C} \cdot\langle a, b\rangle$.

3. $(g, a) \neq 1$ and $(g, b) \neq 1$. Then we have $(g a b, b)=(g a b, a)=1$, which implies that $g=(g a b) \cdot(a b)^{-1} \in \mathfrak{C} \cdot\langle a, b\rangle$.

Since each of these cases leads to a contradiction, we have $G=\mathfrak{C} Y\langle a, b\rangle$.

Let $d \in \mathfrak{C} \backslash H$. Since $\sigma(a d)=a d$, we get $a d=\sigma(a d)=\sigma(a) d$, whence $\sigma(a)=a$, a contradiction. Since $G=\mathfrak{C} \cdot\langle a, b\rangle$, it follows that $G=H=\langle W\rangle$. If $d \in \zeta(G) \cap W$, then $\sigma(a d)=a d$ and $(a d, b)=(a, b) \neq 1$; using Lemma 2 for $\langle a d, b\rangle$, we obtain

$$
-1=f(a d)=f(a) f(d)=f(d) .
$$

Now, we let $\zeta(G) \cap W=\varnothing$ and put $x=a c+b$, where $c \in \mathfrak{C}$. Then there exists $d \in G$ such that $(c, d) \neq 1$, and Lemma 2 implies that $f(g)=1$ for all $g \in G$. Thus, $x^{\sigma}=(a \sigma(c)+b)(a, b)$, and by (1) we have $(\sigma(c)-c)(1-(a, b))=0$. It follows that either $\sigma(c)=c$, or $\operatorname{char}(K)=2$ and $\sigma(c)=(a, b) c$. Therefore, if $\mathfrak{C}$ is Abelian, we obtain statement (ii) of the theorem.

Finally, assume that $\operatorname{char}(K) \neq 2$. Suppose there exist $c, d \in \mathfrak{C}$ such that $(c, d) \neq 1$. If $\sigma(c)=c$, then $f(c)=1$ by what has already been proved, but, by Lemma 2 in $\langle c, d\rangle$, we have $f(c)=-1$, a contradiction. Therefore, $c \in W$ and similarly $d \in W$. We put $x=a c+d$. Clearly, $x^{\sigma}=a c+d(a, b)$ and $(a, b)=1$ by $(1)$, a contradiction. Thus, if $\mathfrak{C}$ is not Abelian, then $\operatorname{char}(K)=2$, and the proof is complete.

Now we are in a position to prove our main theorem.

Proof of the "if" part of the theorem. Set $W=\{w \in G \mid \sigma(w) \neq w\}$ and $H=\langle W\rangle$. If $H$ is Abelian, then, by Lemma 3, statement (i) of the theorem is valid for $G$.

Suppose that $H$ is non-Abelian and that $a, b \in W$ satisfy $(a, b) \neq 1$. By Lemma 4, $G=\langle a, b\rangle \mathrm{Y} \mathfrak{C}=\langle W\rangle$, where $\mathfrak{C}=C_{G}(\langle a, b\rangle)$. If $\mathfrak{C}$ is Abelian, then statement (ii) of our theorem is valid for $G$ by Lemma 4 .

Let $c, d \in C_{G}(\langle a, b\rangle)$ be such that $(c, d) \neq 1$ (i.e., $\mathfrak{C}$ is non-Abelian). By Lemma 4 , we have $\operatorname{char}(K)=2$. If $c, d \in W$, then, by Lemma 4 ,

$$
G=C_{G}(\langle a, b\rangle) \cdot\langle a, b\rangle=C_{G}(\langle c, d\rangle) \cdot\langle c, d\rangle .
$$

Obviously, $C_{G}(\langle a, b\rangle) \cap\langle a, b\rangle \subseteq \zeta(G)$. Therefore, $G$ contains the subgroup $H_{2}=\langle a, b\rangle \mathrm{Y}$ $\langle c, d\rangle$, which cannot be a direct product because $G^{\prime}$ has order 2 .

Since $G^{\prime} \subseteq \mathfrak{R}(G)$, we see that $G / \mathfrak{R}(G)$ is an elementary Abelian 2-group. Let $\tau$ : $G \rightarrow G / \mathfrak{R}(G)=\chi_{i \geq 1}\left\langle a_{i} \mid a_{i}^{2}=1\right\rangle$ be such that $\tau^{-1}\left(a_{1}\right)=a$ and $\tau^{-1}\left(a_{2}\right)=b$. We put $\bar{a}_{i}=\tau^{-1}\left(a_{i}\right)$ for all $i \geq 3$ and $\mathfrak{B}=\left\{a_{i} \mid i \geq 3\right\}$. 
Suppose that for some $s \geq 3$ we have $\left(\bar{a}_{s}, \bar{a}_{i}\right)=1$ for all $i \geq 3$. Such an element is unique, because if $\bar{a}_{t} \neq \bar{a}_{s}$ commutes with all $\bar{a}_{s}$, then $\sigma\left(\bar{a}_{t} \bar{a}_{s}\right)=\bar{a}_{s} \bar{a}_{t}$, whence $a_{s} a_{t}=1$, a contradiction. Put $\mathfrak{B}=\mathfrak{B} \backslash a_{s}, b_{0}=a_{s}, b_{1}=a_{1}$, and $b_{2}=a_{2}$. Note that if such an element $a_{s}$ does not exist, then we put $b_{0}=1$.

Choose $a_{i} \in \mathfrak{B}$. There is $a_{j} \in \mathfrak{B}$ such that $\left(\bar{a}_{i}, \bar{a}_{j}\right) \neq 1$, and we consider the following cases.

1. $\bar{a}_{i}, \bar{a}_{j} \in W$. Put $b_{3}=a_{i}, b_{4}=a_{j}$ and $\mathfrak{B}=\mathfrak{B} \backslash\left\{a_{i}, a_{j}\right\}$.

2. $\quad \bar{a}_{i} \in W$ and $\bar{a}_{j} \notin W$. Clearly, $\left\langle\bar{a}_{1}, \bar{a}_{2}\right\rangle \mathrm{Y}\left\langle\bar{a}_{i}, \bar{a}_{j}\right\rangle \cong\left\langle\bar{a}_{1} \bar{a}_{i}, \bar{a}_{2}\right\rangle \mathrm{Y}\left\langle\bar{a}_{i}, \bar{a}_{2} \bar{a}_{j}\right\rangle$ and $\bar{a}_{1} \bar{a}_{i}, \bar{a}_{2}, \bar{a}_{i}, \bar{a}_{2} \bar{a}_{j} \in W$. Put $b_{1}=\tau\left(\bar{a}_{1} \bar{a}_{i}\right), b_{2}=a_{2}, b_{3}=a_{i}, b_{4}=\tau\left(\bar{a}_{2} \bar{a}_{j}\right)$, and $\mathfrak{B}=$ $\mathfrak{B} \cup\left\{a_{1}, a_{2}\right\} \backslash\left\{b_{1}, b_{2}, b_{3}, b_{4}\right\}$.

3. $\bar{a}_{i}, \bar{a}_{j} \notin W$. Obviously, we have $\bar{a}_{i} \bar{a}_{j} \in W$, so that this case reduces to the preceding one.

Furthermore, if $C_{G}\left(\left\langle\bar{b}_{1}, \bar{b}_{2}\right\rangle \mathrm{Y}\left\langle\bar{b}_{3}, \bar{b}_{4}\right\rangle\right)$ contains a noncommuting pair of elements, then this pair can be chosen in $W$. By continuing this process, we can conclude that $G$ contains a subgroup $\mathfrak{M}=A_{1} \mathrm{Y} A_{2} \mathrm{Y} \ldots$ that is a central product, where each $A_{i}=\left\langle g_{i}, h_{i}\right\rangle$ is a $\sigma$-group and $C_{G}(\mathfrak{M})$ is Abelian. Applying Lemma 4, we arrive at statement (iii) of the theorem, and the proof is complete.

Proof of the "only if" part of the theorem. (i) We can write any $x \in K G$ as $x=x_{1}+x_{2} b$, where $x_{i} \in K H$. Clearly, $x^{\sigma}=x_{1}^{\sigma}+f(b) \sigma(b) x_{2}^{\sigma}=x_{1}^{\sigma}-x_{2} b$ and

$$
x x^{\sigma}=x_{1} x_{1}^{\sigma}-x_{2} x_{2}^{\sigma} b^{2}=x_{1}^{\sigma} x_{1}-x_{2}^{\sigma} x_{2} b^{2}=x^{\sigma} x,
$$

so that $K G$ is a $\sigma$-normal ring.

(ii) Any $x \in K H$ can be written as $x=x_{0}+x_{1} g+x_{2} h+x_{3} g h$, where $x_{i} \in K\left\langle g^{2}, h^{2}, c\right\rangle$ and $c=(g, h)$. Clearly, $x^{\sigma}=x_{0}+\left(x_{1} g+x_{2} h+x_{3} g h\right) c$ and $x x^{\sigma}=x^{\sigma} x$, so that $K H$ is $\sigma$-normal. Suppose that $\sigma(d)=d c$, with $c=(a, b)$. Any $x \in K G$ can be written as $x=\left(w_{0}+u_{1}\right)+\left(w_{2}+u_{3}\right) d$, where $u_{1}=\alpha_{1} a+\alpha_{2} b+\alpha_{3} a b, u_{3}=\beta_{1} a+\beta_{2} b+\beta_{3} a b$, and $\alpha_{i}, \beta_{i}, w_{0}, w_{2} \in K \Re$. Then $x^{\sigma}=\left(w_{0}+u_{1} c\right)-\left(w_{2}+u_{3} c\right) d c$ and $x x^{\sigma}-x^{\sigma} x=$ $\left(u_{3} u_{1}-u_{1} u_{3}\right)(1+c) d$. Since $a b-b a=b a(c-1)$ and $c^{2}=1$, it follows that $x x^{\sigma}-x^{\sigma} x=0$. Thus, $K G$ is $\sigma$-normal. In the case where $\sigma(d)=d$, the proof is similar.

(iii) Put $G_{n}=A_{1} \mathrm{Y} \cdots \mathrm{Y} A_{n}$, where $A_{i}=\left\langle a_{i}, b_{i} \mid c=\left(a_{i}, b_{i}\right)\right\rangle$ is a $\sigma$-subgroup. We use induction on $n$. Any $x \in K G_{n}$ can be written as $x=x_{0}+x_{1} a_{n}+x_{2} b_{n}+x_{3} a_{n} b_{n}$, where $x_{i} \in K\left\langle G_{n-1}, a_{n}^{2}, b_{n}^{2}\right\rangle$. Obviously, $x^{\sigma}=x_{0}^{\sigma}+\left(x_{1}^{\sigma} a_{n}+x_{2}^{\sigma} b_{n}+x_{3}^{\sigma} a_{n} b_{n}\right) c$. Since $K G_{n-1}$ is $\sigma$-normal, we get $x_{i} x_{i}^{\sigma}=x_{i}^{\sigma} x_{i}$ and $x_{i}^{\sigma}(1+c)=x_{i}(1+c)$. The formula

$$
\left(x_{i}+x_{j}\right)\left(x_{i}+x_{j}\right)^{\sigma}=\left(x_{i}+x_{j}\right)^{\sigma}\left(x_{i}+x_{j}\right)
$$

shows that

$$
x_{i} x_{j}^{\sigma}+x_{j} x_{i}^{\sigma}=x_{i}^{\sigma} x_{j}+x_{j}^{\sigma} x_{i} .
$$

Proceeding as in the preceding case, we conclude that

$$
x x^{\sigma}=x^{\sigma} x,
$$

and the proof is complete.

\section{REFERENCES}

[1] S. D. Berman, On the equations $x^{m}=1$ in an integral group ring, Ukrain. Mat. Zh. 7 (1955), no. 3, 253-261. (Russian) MR0077521 (17:1048g)

[2] A. A. Bovdi, Unitarity of the multiplicative group of an integral group ring, Mat. Sb. (N.S.) 119 (161) (1982), no. 3, 387-400; English transl. in Math. USSR-Sb. 47 (1984). MR0678835 (84a:16019)

[3] A. A. Bovdi, P. M. Gudivok, and M. S. Semirot, Normal group rings, Ukrain. Mat. Zh. 37 (1985), no. 1, 3-8; English transl., Ukrainian Math. J. 37 (1985), no. 1, 1-5. MR0780906 (86h:16013)

[4] A. A. Bovdi, Normal twisted group rings, Dokl. Akad. Nauk Ukrain. SSR Ser. A 1990, no. 7, 6-8. (Russian) MR1088083 (91i:20004) 
[5] _ Structure of normal twisted group rings, Publ. Math. Debrecen 51 (1997), 279-293. MR1485224 (98j:16016)

[6] M. Hall, The theory of groups, Chelsea Publ. Co., New York, 1976. MR0414669 (54:2765)

[7] M.-A. Knus, A. Merkurjev, M. Rost, and J.-P. Tignol, The book of involutions, Amer. Math. Soc. Colloq. Publ., vol. 44, Amer. Math. Soc., Providence, RI, 1998. MR1632779 (2000a:16031)

[8] S. P. Novikov, Algebraic construction and properties of Hermitian analogs of K-theory over rings with involution from the viewpoint of Hamiltonian formalism. Applications to differential topology and the theory of characteristic classes. I, II, Izv. Akad. Nauk SSSR Ser. Mat. 34 (1970), 253288; ibid. 34 (1970), 475-500; English transl., Math. USSR-Izv. 4 (1970), 257-292; ibid. 4 (1970), 479-505. MR0292913 (45:1994)

Institute of Mathematics, University of Debrecen, P.O. Box 12, H-4010 Debrecen, Hungary E-mail address: vbovdi@math.klte.hu

Current address: Institute of Mathematics and Informatics, College of Nyíregyháza, Sóstói út 31/b, H-4410 Nyíregyháza, Hungary

Dipartimento di Matematica "E. De Giorgi", Università degli Studi di Lecce, Via ProvinCiAle LeCCE-Arnesano, 73100-LECCE, ItAly

E-mail address: salvatore.siciliano@unile.it

Received 31/AUG/2006

Originally published in English 